\title{
Brain venography performance following the pause of Ad.26.COV2.S COVID-19 vaccine administration
}

\author{
Clayton V. Long ${ }^{1}$. Jonathan D. Clemente ${ }^{2} \cdot$ Sam Singh ${ }^{3} \cdot$ Dale Strong $^{3} \cdot$ Jeremy B. Rhoten $^{4} \cdot$ Tanushree Prasad $^{5}$. \\ Andrew W. Asimos ${ }^{1}$
}

Accepted: 13 October 2021 / Published online: 5 November 2021

(C) The Author(s), under exclusive licence to Springer Science+Business Media, LLC, part of Springer Nature 2021

\begin{abstract}
Cases of cerebral venous thrombosis (CVT) associated with vaccine induced thrombotic thrombocytopenia (VITT) were reported following administration of the adenoviral vector COVID-19 vaccines, resulting in a pause in Ad.26.COV2.S vaccine administration in the United States, beginning on April 14, 2021. We aimed to quantify and characterize an anticipated increase in brain venograms performed in response to this pause. Brain venogram cases were retrospectively identified during the three-week period following the vaccine pause and during the same calendar period in 2019. For venograms performed in 2021, we compared COVID vaccinated to unvaccinated patients. There was a $262 \%$ increase in venograms performed between $2019(n=26)$ and $2021(n=94)$, compared to only a 19\% increase in all radiologic studies. Fifty-seven percent of patients in 2021 had a history of COVID-19 vaccination, with the majority being Ad.26.COV2.S. All patients diagnosed with CVT were unvaccinated. COVID vaccinated patients lacked platelet or D-dimer measurements consistent with VITT. Significantly more vaccinated versus unvaccinated patients had a headache $(94 \%$ vs $70 \%, p=0.0014)$, but otherwise lacked compelling CVT presentations, such as decreased/altered consciousness ( $7 \%$ vs $23 \%, \mathrm{p}=0.036)$, neurologic deficit $(28 \%$ vs $48 \%, \mathrm{p}=0.049)$, and current/recent pregnancy ( $2 \% \mathrm{vs} 28 \%, \mathrm{p}=0.0003)$. We found a dramatic increase in brain venograms performed following publicity of rare COVID-19 vaccine associated CVT cases, with no CVTs identified in vaccinated patients. Clinicians should carefully consider if brain venogram performance is indicated in COVID-19 vaccinated patients lacking thrombocytopenia and D-dimer elevation, especially without other compelling CVT risk factors or symptoms.
\end{abstract}

Keywords Venogram $\cdot$ Ad.26.COV2.S $\cdot$ COVID-19 $\cdot$ Vaccine $\cdot$ CVT $\cdot$ Thrombosis

\section{Highlights}

Andrew W. Asimos

andrew.asimos@atriumhealth.org

1 Department of Emergency Medicine, Medical Education Building, Atrium Health's Carolinas Medical Center, 1000 Blythe Blvd, Charlotte, NC 28203, USA

2 Charlotte Radiology, Charlotte, PANC, USA

3 Atrium Health, Information and Analytics Services, Charlotte, NC, USA

4 Atrium Health, Neurosciences Institute, Charlotte, NC, USA

5 Clinical Analytics, Atrium Health, Charlotte, USA
- A dramatic increase in venogram performance occurred during the three-week period following the 2021 pause in Ad.26.COV2.S COVID-19 vaccine administration compared to the same period in 2019.

- The majority of patients undergoing venography had a history of COVID-19 vaccination, most of whom had received the Ad.26.COV2.S vaccine.

- All patients diagnosed with CVT following the vaccine pause were unvaccinated.

- Only one patient undergoing venography was thrombocytopenic and a small minority of patients had a D-dimer performed, with no patients meeting laboratory criteria for VITT.

- This study suggests that the observed increase in venogram utilization occurred in a population at low risk for 
Table 1 Comparison of Venogram and All Radiologic Studies Performed during each three-week Study Period

\begin{tabular}{llll}
\hline Number of studies by year & 2019 & 2020 & 2021 \\
\hline Venograms & 26 & 18 & 94 \\
All Radiologic Studies & 98,406 & 59,094 & 117,335 \\
Proportions of Venograms & $0.026 \%$ & $0.030 \%$ & $0.080 \%$ \\
\hline Relative change in studies between years & $2019-2020$ & $2020-2021$ & $2019-2021$ \\
\hline Venograms & $-31 \%$ & $422 \%$ & $262 \%$ \\
All Radiologic Studies & $-40 \%$ & $99 \%$ & $19 \%$ \\
Proportions of Venograms & $-15 \%$ & $163 \%$ & $203 \%$ \\
\hline
\end{tabular}

The top portion of the table describes the total number of venograms and all radiologic studies for each study period. All radiologic studies include any performed within the 12 hospitals, 7 free-standing emergency departments, and 7 outpatient imaging centers included in this study

The bottom portion of the table describes the relative change in venograms and all radiologic studies between all three study years

CVT; therefore, in patients recently vaccinated with an adenoviral vector COVID-19 vaccine, in the absence of thrombocytopenia and D-dimer elevation, especially without other compelling CVT risk factors or symptom it may be prudent to reconsider pursuing venography.

\section{Introduction}

In April 2021, several cases of cerebral venous thrombosis (CVT) were reported following administration of the Ad.26.COV2.S COVID-19 vaccine (Janssen Biotech, Inc., a Janssen Pharmaceutical company, Johnson \& Johnson) and ChAdOx1 nCoV-19 vaccine (Oxford/AstraZeneca) [1-3]. As a result, on April 14, 2021, the U.S. Food and Drug Administration (FDA) and U.S. Centers for Disease Control and Prevention (CDC) announced a "pause" in the use of the single-dose Ad.26.COV2.S vaccine while an investigation was conducted into the rare reports of CVT $[4,5]$. As of April 14th, our large healthcare system had administered approximately 18,500 doses of the Ad.26.COV2.S vaccine, while over 6.8 million doses had been administered throughout the U.S [6]. Because of the widespread attention the rare CVT events received, and because CVT can be challenging to diagnose clinically $[7,8]$, we sought to quantify and characterize the patients undergoing brain venography to evaluate for CVT in the three weeks immediately following this announcement.

\section{Methods}

To determine the difference in brain venogram utilization following the pause compared to historical cohorts, the total number of venograms performed from April 14 to May 5 in 2019 (pre-pandemic) and 2020 (pandemic) were compared to the same three-week period in 2021. The 2021 cohort was divided into vaccinated versus unvaccinated patients for comparative analyses. Cases were identified in a retrospective cross-sectional manner based on the inclusion criteria of age 18 and older who had a computed tomography brain venogram (CTV) or magnetic resonance brain venogram (MRV) performed at one of our healthcare system's 12 hospitals, 7 free-standing emergency departments, and 7 outpatient imaging centers, covering a region with a population of approximately 1.9 million people. Patients less than 18 years of age and any patient with health records not accessible via our electronic medical record (EMR) were excluded from the study. We additionally excluded patients who had a repeat venogram performed to assess progression or resolution of a previously diagnosed CVT. Individual cases were identified by querying the radiology report database (Primordial, Prism, Nuance Communications, Burlington, MA) for our healthcare system. Keyword search of the radiology reports was employed using the following keywords: venogram, venography, CTV, MRV, venous sinus thrombosis, venous sinus thrombus. Data abstracted from our EMR included patient demographics, CVT symptoms, CVT risk factors, comorbidities, radiologic evaluation, and additional nonimaging diagnostic workup. Vaccination status was verified by accessing an enterprise data warehouse, which facilitated capturing the vaccination record for patients vaccinated outside our healthcare system. We analyzed data with standard statistical methods utilizing the SAS program version 9.4 (SAS, Cary, NC, USA). Descriptive statistics are reported as means with corresponding standard deviations for continuous variables and percentages for categorical variables. Univariate analysis was performed to compare the vaccinated versus unvaccinated cohorts. Categorical variables were evaluated using Pearson's chi-squared and Fisher's exact test where appropriate. Continuous and ordinal variables were evaluated using Wilcoxon two-sample tests. Statistical significance was set at $p \leq 0.05$, and all reported $p$ values are two-tailed. 
Table 2 Comparison Based on Vaccination Status for Patients with Brain Venograms Performed

\begin{tabular}{|c|c|c|c|}
\hline \multirow[t]{2}{*}{ Variable } & \multicolumn{2}{|c|}{2021 COVID-19 Vaccination } & \multirow[t]{2}{*}{ p-value } \\
\hline & No $(n=40)$ & Yes $(n=54)$ & \\
\hline \multicolumn{4}{|l|}{ Demographics } \\
\hline Age, mean $\pm S D$ & $40.2 \pm 16.6$ & $43.3 \pm 12.5$ & 0.147 \\
\hline Female Sex & $31(77.5)$ & $48(88.9)$ & 0.136 \\
\hline \multicolumn{4}{|l|}{ Race } \\
\hline White or Caucasian & $23(57.5)$ & $33(61.1)$ & \multirow[t]{7}{*}{0.242} \\
\hline Black or African American & $12(30.0)$ & $18(33.3)$ & \\
\hline Asian & $1(2.5)$ & $1(1.9)$ & \\
\hline American Indian or Alaska native & $0(0.0)$ & $0(0.0)$ & \\
\hline Hispanic or Latino & $0(0.0)$ & $2(3.6)$ & \\
\hline Middle Eastern or North African & $2(5.0)$ & $0(0.0)$ & \\
\hline Not specified & $2(5.0)$ & $0(0.0)$ & \\
\hline \multicolumn{4}{|l|}{ Ethnicity } \\
\hline Hispanic or Latino & $1(2.5)$ & $3(5.6)$ & \multirow[t]{3}{*}{0.625} \\
\hline Non-Hispanic or Latino & $38(95.0)$ & $48(88.9)$ & \\
\hline Not specified & $1(2.5)$ & $3(5.6)$ & \\
\hline $\mathrm{BMI}$, mean $\pm \mathrm{SD}$ & $32.0 \pm 8.4$ & $31.4 \pm 9.2$ & 0.701 \\
\hline \multicolumn{4}{|l|}{ Symptoms } \\
\hline Headache & $28(70.0)$ & $51(94.4)$ & 0.001 \\
\hline Decreased/Altered Consciousness & $9(22.5)$ & $4(7.4)$ & 0.036 \\
\hline Neck Pain & $3(7.9)$ & $0(0.0)$ & 0.085 \\
\hline Neurologic Deficit & $19(47.5)$ & $15(27.8)$ & 0.049 \\
\hline Seizure & $3(7.7)$ & $0(0.0)$ & 0.070 \\
\hline Vomiting & $6(15.0)$ & $3(5.6)$ & 0.163 \\
\hline \multicolumn{4}{|c|}{ Prothrombotic Risk Factors and Other Comorbidities } \\
\hline Current or recent pregnancy & $11(27.5)$ & $1(1.8)$ & 0.0003 \\
\hline Prothrombotic Condition & $3(7.5)$ & $4(7.5)$ & 1.000 \\
\hline Migraine history & $9(22.5)$ & $12(22.2)$ & 0.975 \\
\hline Head trauma within the previous month & $5(12.5)$ & $1(1.8)$ & 0.080 \\
\hline Neurosurgery within the previous month & $3(7.7)$ & $0(0.0)$ & 0.070 \\
\hline Hospitalization within the previous month & $9(22.5)$ & $1(1.8)$ & 0.002 \\
\hline CNS/Ear infection & $2(5.0)$ & $1(1.8)$ & 0.571 \\
\hline CNS Malignancy & $0(0.0)$ & $0(0.0)$ & - \\
\hline Malignancy other & $0(0.0)$ & $1(1.8)$ & 1.000 \\
\hline OCP Use/Hormone Replacement Therapy & $3(7.7)$ & $8(14.8)$ & 0.348 \\
\hline Current Smoking & $9(22.5)$ & $9(16.7)$ & 0.477 \\
\hline Previous CVT & $2(5.0)$ & $0(0.0)$ & 0.178 \\
\hline \multicolumn{4}{|l|}{ Imaging and Diagnostic Studies Performed } \\
\hline CVT on imaging & $2(5.0)$ & $0(0.0)$ & 0.178 \\
\hline Platelet count performed & $35(87.5)$ & $45(83.3)$ & 0.575 \\
\hline Platelet count, Mean \pm SD & $265.0 \pm 73.0$ & $262.1 \pm 76.0$ & 0.779 \\
\hline D-dimer performed & $1(2.5)$ & $3(5.6)$ & 0.364 \\
\hline
\end{tabular}

The number of cases per year identified for each categorical variable are represented with the percentage of total cases for that year, $\mathrm{n}(\%)$

$S D$ standard deviation, $B M I$ body mass index, $C N S$ central nervous system, $O C P$ oral contraceptive pill, $C V T$ cerebral venous thrombosis

${ }^{\text {a }}$ Prespecified as previous deep venous thrombosis or pulmonary embolism, Deficiencies of protein C, protein S, antithrombin III, plasminogen, activated protein C resistance/factor V Leiden mutation, anticardiolipin antibodies, lupus anticoagulant 


\section{Results}

There was a $262 \%$ increase in the number of venograms performed when comparing the same three-week period from $2021(n=94)$ following the vaccine pause to $2019(n=26)$. In contrast, there were 117,335 radiologic studies performed during the 3 -week period in 2021, versus 98,406 in 2019, representing only a $19 \%$ increase in all radiologic studies performed. Additional relative changes of venograms and all radiologic studies performed in 2019, 2020, and 2021 are listed in Table 1. For patients undergoing venography in $2021,56 \%(n=53 / 94)$ of venograms were performed in the ED, $32 \%(n=30 / 94)$ were performed as an inpatient, and $12 \%(n=11 / 94)$ were ordered as outpatient studies. Fifty-seven percent $(n=54)$ of patients had a history of COVID-19 vaccination, with the majority being Ad.26. COV2.S vaccinations. A comparison of COVID-19 vaccinated versus unvaccinated patients undergoing venography in our three-week study period is provided in Table 2. Significantly more vaccinated versus unvaccinated patients had a headache $(94 \%$ vs $70 \%, p=0.001)$, but otherwise lacked compelling presentations for CVT, such as decreased/altered consciousness ( $7 \%$ vs $23 \%, \mathrm{p}=0.036$ ), neurologic deficit ( $28 \%$ vs $48 \%, p=0.049)$, and current/recent pregnancy $(2 \%$ vs $28 \%, \mathrm{p}=0.0003$ ). No COVID-19 vaccinated patients were diagnosed with a CVT, versus $5 \%(n=2)$ in the unvaccinated group. Of COVID-19 vaccinated patients undergoing venography, $83 \%(n=45)$ had a platelet count performed, of whom only one had thrombocytopenia, defined as $<150 \times 10^{9} / \mathrm{L}$. Additionally, only $5.6 \%(n=3)$ of patients had a D-dimer performed, with no values markedly elevated and consistent with VITT ( $>4$ times upper limit of normal).

\section{Discussion}

Our data suggest that during the same three-week period in 2019, 2020, and 2021 that trends in venogram utilization likely resulted from the reported rare risk of CVT associated with COVID-19 vaccination, and not from the risk of CVT associated with COVID infection. Moreover, the increased venogram utilization in vaccinated patients appears to have been in a population at overall low risk for CVT, because they lacked compelling symptoms for CVT (beyond headache), lacked prothrombotic risk factors or other comorbidities predisposing to CVT, and lacked laboratory findings consistent with VITT. Given the dramatic increase we found in venograms performed within just one healthcare system that was associated with the extensive publicity of rare CVT cases in the setting of VITT, it is likely venogram utilization increased in other settings as well. Moving forward, clinicians should carefully consider if performance of a venogram is indicated in adenoviral vector COVID-19 vaccinated individuals, in the absence of thrombocytopenia and D-dimer elevation, especially without other compelling CVT risk factors or symptoms.

Funding None.

Data availability Data available within the article or its supplementary material.

Code availability Not applicable.

\section{Declarations}

Conflict of interest None.

Ethical approval Electronic medical record access approved by institutional review board.

\section{References}

1. Schultz NH, Sørvoll IH, Michelsen AE, Munthe LA, LundJohansen F, Ahlen MT et al (2021) Thrombosis and thrombocytopenia after ChAdOx1 nCoV-19 vaccination. N Engl J Med. https://doi.org/10.1056/NEJMoa2104882

2. Greinacher A, Thiele T, Warkentin TE, Weisser K, Kyrle PA, Eichinger S (2021) Thrombotic thrombocytopenia after ChAdOx1 nCov-19 vaccination. N Engl J Med. https://doi.org/10.1056/ NEJMoa2104840

3. Muir K-L, Kallam A, Koepsell SA, Gundabolu K (2021) Thrombotic thrombocytopenia after Ad26.COV2.S vaccination. N Engl J Med. https://doi.org/10.1056/NEJMc2105869

4. CDC Health Alert Network. Cases of Cerebral Venous Sinus Thrombosis with Thrombocytopenia after Receipt of the Johnson \& Johnson COVID-19 Vaccine. The Centers for Disease Control and Prevention (CDC), 2021.

5. Sadoff J, Davis K, Douoguih M (2021) Thrombotic thrombocytopenia after Ad26.COV2.S vaccination-response from the manufacturer. N Engl J Med. https://doi.org/10.1056/NEJMc2106075

6. Shay DK, Gee J, Su JR et al (2021) Safety monitoring of the Janssen (Johnson \& Johnson) COVID-19 vaccine-United States, March-April 2021. Morb Mortal Wkly Rep 70(18):680-684

7. Idiculla PS, Gurala D, Palanisamy M, Vijayakumar R, Dhandapani S, Nagarajan E (2020) Cerebral venous thrombosis: a comprehensive review. Eur Neurol. https://doi.org/10.1159/000509802

8. Capecchi M, Abbattista M, Martinelli I (2018) Cerebral venous sinus thrombosis. J Thromb Haemost 16:1918-1931

Publisher's Note Springer Nature remains neutral with regard to jurisdictional claims in published maps and institutional affiliations. 